found wanting in spite of a timely employment. And then it will go the same way the other inoculations and vaccinations for syphilis, anthrax, cholera, hydrophobia and tuberculosis went. Yet I want to say that I am far from thinking that those industrious investigations which have been and are being made concerning the serum-therapy, are in vain. On the contrary, they will be of great benefit to mankind, for they will finally convince us that we can not rely upon the blood serum of a horse or any other animal, not even on that of another man, but only upon our own individual serum.

And they will teach us that only by a hygienic way of living can we immunize ourselves; that means, improve the physiologic functions of our body and the bactericide power of our blood. They will also teach us that in disease we can rely upon the same agents only, on which natural immunization is based and of which physiologic treatment consists.

643 W. 12th Street.

\section{GENERAL SEPTIC PERITONITIS TREATED WITH SEPTICEMIC SERUM. \\ BY W. A. MACFARLANE, M.D. CHICA GOO.}

Mrs. H., age 30; general health fairly good; eight years ago gave birth at the eighth month to a healthy child; it lived three days. She became pregnant in about one year and at end of third month miscarried. About five years ago was called to see her for the first time. On examination found os dilated to some extent, with double laceration of cervix: most extensive on left side. Found patient had had considerable hemorrhage. Decided to prevent miscarriage if possible, as they were anxious to have children. Succeeded in stopping the hemorrhage to some extent before leaving. Was called the next morning in great haste; found her considerably prostrated from loss of blood and flowing profusely. Decided at once to dilate and deliver, which was done as rapidly as possible, but not until she was very much prostrated, being obliged to use a number of hypodermic injections of digitalin, strych. sulph., glonoin and brandy. Found great difficulty in removing all of the placenta as it was considerably adherent. Left her resting well and heart acting as well as could be expected. Called next morning and curetted, removing some small pieces of membrane, washed out uterus with sol. bichlorid, 1 to 2,000, and repeated several days, patient making fairly good recovery.

Treated her after for some time, getting her in general good health and advised her to submit to an operation for lacerated cervix, as I decided that would be the only hope of her ever going through to full term. Could not find any hereditary taint as far as could be ascertained, although could not get perfectly clear history, as she was very young when leaving Europe. She continued in very excellent health for over two years and became pregnant again, continuing so without any apparent danger until end of seventh month, when I was called suddenly and found her in true labor; delivered her of a large, healthy child, apparently perfect in every way. Used every means possible to save the child; died the fifth day. Patient made good recovery and became in good health again and continued so up to Jan. 3, 1896, being two years since last premature labor and enjoying as good health as could be desired, except occasional visits to my office complaining of some slight stomach derangement. January 3, was called and on examination found os: slightly dilated with considerable hemorrhage. Prescribed tinct. opii, gtt. $x$ in water every three to. four hours; visited her at 9 P.M., hemorrhage slight, resting quietly, without any pain. January 4,10 . A.M., patient stated to me that at 5 A.M. she had hacl profuse hemorrhage and strong pains from 5 to 6 and that everything had come away. On my making. examination found that there were still some placental remains. Removed a large piece, all that I could find; concluded that I had removed all. January 5, 9 A.M., temperature 99.6, pulse 90, hemorrhage normal, patient stating that she was feeling well, taking considerable nourishment and had rested well during the night. Examined again for placental remains, found os still slightly open, but could not find any indication of remaining membrane. January 6,9 A.M., temperature 100, pulse 96; patient had rested well during night, taking considerable nourishment, principally milk. Discharge normal. January 7, 10 A.M., found patient not so well; temperature 102, pulse 108 . Gave her intra-uterine injection sol. bichlorid 1 to 2,000 and prescribed salophen and phenacetin $2 \frac{1}{2}$ grs. each in one powder every three hours; tr. nuc. vom., $3 \mathrm{~m}$.; diyitalin, gr. 1-80; tr. capsica, 2 m.; quinia sulph., 2 gr. in syr., one teaspoonful every three hours; brandy every two hours. Called at 9 P.M., temperature 101.5, pulse 102 , sweating freely. Was called called at 2 A.M. by husband, stated over telephone that patient was in very severe pain and had chills and pains over lower portion of abdomen. Ordered druggist to put up morph. sulph. $1 \mathrm{gr}$, and divide into four powders, to be given every three or four hours in a little water. Flaxseed meal, stramonii fol. in hot poultice to be applied over pelvis. January 8 visited patient at 8 A.M., and found her suffering severe pains; temperature 105.4, pulse 132 and thready, and in profuse perspiration; features pinched and sallow and frightened expression of face, loss of all hope, marked tympanites over whole of abdomen, complaining of the pain being most severe and extending up sides of abdomen, vomiting continuously; had not been able to retain anything on stomach since $4 \mathrm{~A}$.M., not even a teaspoonful of water; small pellets of ice being dissolved in mouth would cause severe retching. Decided to curette at once, which was done, assisted by Dr. P. T. Burns, getting considerable broken-down tissue, ollor very great. Washed out uterus with as hot water as could be borne, mopping out uterus with pledgets of absorbent cotton and applying to inner surface of womb sol. equal parts carbolic acid and iodin and placing over external part absorbent cotton pad; applied poultices of flaxseed meal and stramonii fol., which relieved pain to some extent. Prescribed small doses of dilute phos. acid in a little water at frequent intervals, and bromo-caf. to allay irritation of stomach, ordering small doses of mag. calc. as soon as condition of stomach would permit. Did not attempt to give anything in way of medicine or nourishment for several hours and then began with small amounts of liq. peptonoids and champagne. In fact, she was not able to retain anything of any consequence on the stomach for the next twenty-four hours. I telephoned at once to Truax \& Co. for septicemic serum, Dr. Burns agreeing with me that there was not the slightest hope for her unless there might be some benefit to be derived through the aid of septicemic treatment and we had at that time but little faith in it. I 
secured the serum at 7 P.M. and gave her 20 c.c.; tem. and carbolic acid and irrigating the uterine cavity the perature still over 105, pulse 130, and sweating pro- temperature did not vary one degree, but the first fusely; vomiting very severe; occasionally retaining injection of serum brought all of the symptoms below a little champagne and water in teaspoonful doses. the danger line in twelve hours and quieted the exSymptoms but slightly, if any, improved from morning. treme irritation of the stomach, enabling it to retain Remained until 9 oclock. At that time temperature medicines and nourishment, which we had not been 105.6, pulse about the same as at 7 P.M. January 9, able to do, in twenty-four hours from 4 A.M., January 9 A.u., was very much surprised on examination to 9 , to about the same hour January 10, twelve hours find such marked improvement: temperature slightly less than 102, pulse 112, stronger and quite full; pain very much lessened with less tympanites; had been able to retain medicines, stimulants and some considerable nourishment: had slept possibly two or three hours during the early morning, being disturbed, as she stated, by bad dreams; gave her the remaining 5 c.c. and procured another bottle 25 c.c., and gave another injection, 10 c.c., same evening. January 10, 9 A.M., patient much improved in every way; temperature 101.5, pulse slower, stronger and more compressible, perspiration free, pain slight, tympanites lessened; had not vomited for twenty-four hours, but had occasional slight nausea. Returned at 8:30 P.M. and gave 10 c.c., making 40 c.c. in all; temperature 101.5, pulse improving, patient hopeful, pinched expression of face and sallowness less marked; stomach could now retain all nourishments, oatmeal gruel, buttermilk, sweet milk, broths and beef peptonoids, etc. Pushed medicines that had been set aside since beginning of nausea, which was gradually diminished as she improved from day to day, until dose three times a day was given. Jimuary 11, 9 A.M., temperature 101, pulse 108 , stronger and fuller, still sweating profusely, especially while sleeping; rests fairly well, but broken by dreams; stomach in good condition, taking large quantities of nourishment; does not suffer pain except occasionally; hot poultices still applied every three or four hours to the abdomen: and intra-uterine injections of hot water given every day (10 to 12 quarts); sol. of bichlorid 1 to 2,000 ( 2 quarts) given immediately after. January 12,9 A.M., temperature 102 , pulse more rapid; otherwise apparently doing as well as when I saw her the morning previous. January 13, temperature and pulse as on the 12th. January 14 , temporature 100.5 , pulse 104 . Temperature after this date never rose above 101 at any one time, but usually remained between 99 and 100 for several days, heart apparently showing the effect of the sepsis longer. January 16, temperature 99; patient states that she feels well but very weak. Asks to be allowed to sit up; sweating lessening each day; has been given alcohol baths twice a day; still sweats considerable while sleeping: her rest is not now so much disturbed by dreams. Removed poultice and gave an alcohol bath. Two thicknesses of cotton wool placed over whole of abdomen and fastened by wide bandage, drawn tightly over lower portion. January $17,10 \mathrm{~A}$. M., she sat up about an hour: temperature about normal; complains of weakness, stimulants and tonics have been given freely, taking large quantities of milk and gruel, buttermilk principally, sufficient amount of mag. calc. to keep bowels active, has been given throughout the course of the disease. Kidneys have acted well since stomach being enabled to retain fluids. January 20 visited her for the last time; temperature normal, pulse 90; sitting up severul times each day.

Summary. - This case undoubtedly demonstrites the efficacy of the serum in cases of septic peritonitis. Even after thorough curetting, swabbing with iodin after injection of serum. It also produced continuous and profuse perspiration. This latter condition was without doubt instrumental in draining the system of vast quantities of sepsis and being now enabled to take in unlimited quantities of fluids; there was no special drain on the vitality of the patient. Thorough action of kidneys and bowels, no doubt, also assisted in the process of elimination.

Another great feature in this treatment is the surprisingly rapid convalesence of the patient. No sooner was the infection conquered when the patient appeared to be at death's door than within twenty-four hours the change was from a dying woman to one asking to sit up. Had her heart been in relation to her temperature I could have discharged her as cured three days earlier.

\title{
REPORT OF RESULTS AND RECOVERIES OBTAINED BY THE USE OF ANTI- TUBERCLE SERUM.
}

\author{
BY A. M. HAYDEN, M.D. \\ CHIEF SURGEON ST. MARY'S HOSPITAL. \\ EVANSVILLE, IND.
}

The results I have obtained from the use of Paul Paquin's Antitubercle Serum are as follows:

Case 1.-My first case was one with large cavities in left lung, hepatization of the lower portion of right lung; had a great many hemorrhages, sputum contained large quantities of bacilli ; weight 120 pounds. Treatment commenced May 10, 1895. In three months tubercle bacilli disappeared from sputum entirely, and it has remained free from them ever since. The cavities in left lung healed up, with great contraction of the chest on that side, three or four inches, which has remained so. The patient ceased to expectorate heavy sputa. Has had no hemorrhages since treatment began. He has weighed 140 pounds for the last six months; rides a bicycle, eats well and commences his duties as assessor to-morrow. After the first three months, I gave him from one to two ounces, only at intervals of one and two months. I am satisfied that he would have been dead long before this if he had not had the serum.

Case 2.-A young man 25 years old. Family history was that of tuberculosis. His sputum contained quantities of tubercular bacilli ; weight 110 pounds : had been West without being benefited. He had night sweats, fever and all symptoms of the dread disease. I commenced his treatment Sept. 8, 1895. Gave him 56 daily injections of $35 \mathrm{~ms}$. each. Tubercular bacilli disappeared from sputum and he gained ten pounds in weight. January $20 \mathrm{I}$ commenced again and gave him 16 more injections, during which time he gained five pounds, which is more than he had ever weighed in his life. He is still in splendid health and says he is feeling better than he ever did. I advised him, to-day, to take another ounce of the serum, to guard against relapses.

Ca.se 3.-A young man with long standing hip.joint disease. I gave him one ounce in January, 1896, during which time he gained sixteen pounds and improved greatly in general health. He then stopped for two weeks, during which time he ceased to gain in flesh. He again commenced taking the serum, taking one ounce more, and gained ten pounds. His health has been much improved since.

Case 4.-Patient was a man aged 40. Several brothers had died of tuberculosis. He was taken sick in November, 1895, with cough, night sweats and fever. His family physician treated him until January, 1896, when I was called. He was much emaciated, no appetite, had night sweats, fever, etc. While he coughed almost incessantly, he did not expectorate. I failed to find any lesion of lungs in physical examination. 